\title{
¿Existe la medicina sin pacientes?
}

\author{
ALbert J. Novell \\ Presidente Foro Español de Pacientes. Director General Fundació Biblioteca Josep Laporte \\ Profesor Asociado Facultad de Medicina y Cirugía Universitat Autònoma de Barcelona
}

\section{DECLARACIÓN DE BARCELONA DE LAS ASOCIACIONES DE PACIENTES}

En mayo de 2003 representantes de 50 organizaciones de pacientes y usuarios de la sanidad se reunieron en Barcelona y mediante metodología cualitativa de grupos focales estructuraron un Decálogo de demandas específicas a los servicios de salud. Este Decálogo se conoce como la Declaración de Barcelona de las Asociaciones de Pacientes (www.webpacientes.org) y la promoción del mismo ha dado lugar a la creación del Foro Español de Pacientes (Tabla 1). La implantación de la Declaración de Barcelona debería introducir cambios en la formación y determinación de competencias en los profesionales de la salud. El cambio más importante conlleva contemplar al paciente como un sujeto enfermo más que como una enfermedad objeto de estudio. Ello supone reconocer que, además de un modelo de paciente pasivo, existe un tipo de enfermo más activo y que, por lo tanto, el modelo de relación médico-paciente paternalista debe ser reemplazado por uno más deliberativo.

Tabla 1. Declaración de Barcelona

1. Información de calidad contrastada respetando la pluralidad de las fuentes.

2. Decisiones centradas en el paciente.

3. Respeto a los valores y a la autonomía del paciente informado.

4. Relación médico-paciente basada en el respeto y en la confianza mutua.

5. Formación y entrenamiento específico en habilidades de comunicación para profesionales.

6. Participación de los pacientes en la determinación de prioridades en la asistencia sanitaria.

7. Democratización formal de las decisiones sanitarias.

8. Reconocimiento de las organizaciones de pacientes como agentes de la política sanitaria.

9. Mejora del conocimiento que tienen los pacientes sobre sus derechos básicos.

10. Garantía de cumplimiento de los derechos básicos de los pacientes.
Los cinco primeros principios de la Declaración de Barcelona requieren el desarrollo de estrategias de formación médica, a nivel de pregrado y posgrado, orientadas a la adopción de una relación médico-paciente más simétrica. Los profesionales de la salud, sobre todo los médicos que asisten a pacientes, deberían estar capacitados para identificar los valores de los enfermos y reconocer, a partir de los mismos, hasta que punto éstos prefieren implicarse en las decisiones que afectan a su salud. El modelo actual de relación médico-paciente contempla la existencia de una gran variedad de actitudes y comportamientos, dependiendo éstos de las personas y las circunstancias. Así, hay pacientes que prefieren no saber mucho sobre su enfermedad y, por lo tanto, dejar que sean los profesionales los que tomen decisiones por ellos, y existen otros enfermos que quieren saber y decidir con sus médicos cuál es el tratamiento más apropiado. Esta coexistencia de diferentes tipos de pacientes y de diversas situaciones clínicas amplia el abanico de competencias de los profesionales de la salud e incrementa las demandas de los pacientes por una mejor comunicación médico-paciente. La formación en habilidades de relación y comunicación de los profesionales y una buena educación de los pacientes constituyen uno de los retos más importantes de los sistemas sanitarios modernos.

Otro elemento importante en la determinación de las competencias de los médicos del siglo XXI es la valoración de su perfil por parte de los pacientes. En otras palabras, para que la profesión médica responda a las necesidades de la sociedad se debe preguntar a los ciudadanos cómo quieren que sean sus médicos y qué es lo que más valoran de su perfil competencial. Las asociaciones de pacientes pueden jugar un papel muy importante, junto con las universidades, colegios profesionales y sociedades científicas, en la valoración de las competencias de los profesionales y del desempeño profesional. La introducción de unas competencias profesionales más sensibles a la realidad actual del paciente mediante las pruebas ECOE y la participación del Foro Español de Pacientes en la determinación del portafolio de la especialidad de medicina familiar y 
comunitaria llevada a cabo por la SEMFYC, son ejemplos de una valoración social, y no exclusivamente profesional y basada en expertos, de las competencias de los médicos. Estas estrategias responderían de forma adecuada a los cinco últimos principios de la Declaración de Barcelona, donde destaca el reconocimiento y el cumplimiento de los derechos básicos de los pacientes.

\section{EL MANIFIESTO DE MADRID DEL DÍA MUNDIAL DEL ASMA}

La sanidad está experimentando en la actualidad un cambio social sin precedentes en la historia de la humanidad. La rapidez del cambio supera la capacidad de los profesionales para asimilar las consecuencias del mismo. Uno de los elementos claves de este cambio viene promovido por la aparición del nuevo modelo de paciente activo expuesto en el anterior apartado. Un ciudadano con un nivel educativo más elevado, el acceso universal a información sanitaria mediante Internet, la definición de la salud como un bien individual, además de colectivo, y la aparición de las asociaciones de pacientes como intermediarios en la relación entre agentes sanitarios y pacientes son, entre otros factores, lo elementos que determinan una mayor conciencia y conocimiento en los pacientes sobre sus derechos y la responsabilidad asociada al hecho de estar enfermo.

El verbo enfermar se conjuga en primera persona en todos los tiempos verbales y la experiencia que se deriva de esa vivencia pertenece a los pacientes. Combinar las experiencias de los pacientes y las de los profesionales permite identificar necesidades no satisfechas y oportunidades de mejora en el manejo clínico de condiciones clínicas específicas. El Manifiesto de Madrid del Día Mundial del Asma surgió de una reunión conjunta de profesionales de la salud implicados en el cuidado de los enfermos asmáticos con representantes de las asociaciones de pacientes (Tabla 2). Las propuestas establecidas en ese Manifiesto deberían permitir un tratamiento más efectivo, equitativo y eficiente de los pacientes afectados por la enfermedad asmática. La realidad actual del Sistema Nacional de Salud debería promover una mayor implicación de los pacientes en la definición de los objetos terapéuticos de interés, lo que contribuiría a una mejora en la adherencia a los planes terapéuticos. En el caso del asma se estima que un $60 \%$ de los pacientes no cumplen con la terapia prescrita, por lo que cabría preguntarse si una mayor implicación de los pacientes conseguiría dis- minuir el elevado fracaso terapéutico. Es por ello que hay que tener en cuenta al paciente. Sin pacientes, quizás no existiría la medicina.

Tabla 2. Manifiesto de Madrid del Día Mundial del Asma

1. Definir objetivos terapéuticos que, además de contemplar el tratamiento integral del asma y sus síntomas, sean compatibles con una vida cotidiana normal y respondan a las necesidades expresadas por los pacientes.

2. Establecer un plan nacional de prevención y control del asma que aborde el manejo de la condición clínica desde una perspectiva global, tanto como problema de salud pública como en su dimensión clínica.

3. Desarrollar estrategias que promuevan un mayor cumplimiento terapéutico por parte de los pacientes con el tratamiento.

4. Elaborar guías de práctica clínica actualizadas y de calidad científica acreditada que permitan a los profesionales su traslación a planes terapéuticos personalizados, adaptados a las características de los pacientes.

5. Favorecer la coordinación entre niveles asistenciales, entre especialidades y entre profesionales, definiendo la atención sanitaria del paciente asmático como una estrategia asistencial integral y multidisciplinar que precisa la implicación del paciente.

6. Diseñar estrategias educativas que permitan una mejor comunicación entre profesionales y pacientes.

7. Establecer estrategias que permitan evaluar la calidad asistencial proporcionada a los pacientes afectados por asma, así como el cumplimiento de los objetivos terapéuticos de prevención y manejo adecuado de la enfermedad.

8. Facilitar un mayor conocimiento sobre estrategias de autocuidado y de control de la enfermedad por parte de los pacientes y sus familiares.

9. Aumentar la sensibilización de la población y de las Administraciones públicas sobre las necesidades no atendidas adecuadamente en el manejo clínico y social del asma.

10. Promover la agrupación de las asociaciones de pacientes existentes en España en el seno de una federación de asociaciones.

Agradecimientos:

A Maria Dolors Navarro-Rubio, Laura Fernández y Enric Pineda por su colaboración en la Declaración de Barcelona y en el Manifiesto de Madrid del Día Mundial del Asma.

La Declaración de Barcelona de las Asociaciones de Pacientes y el Manifiesto de Madrid del Día Mundial del Asma han sido posibles gracias a la colaboración de múltiples asociaciones de pacientes y de profesionales. Los dos proyectos han recibido la financiación independiente de MSD España. 